\title{
Holistic Psychopharmacology and Promiscuous Plants and Principles of Ayurveda
}

\author{
Shyam Sunder Chatterjee ${ }^{1}$, Vikas Kumar ${ }^{2 *}$ \\ ${ }^{1}$ Stettiner Str. 1, Karlsruhe, Germany; ${ }^{2}$ Neuropharmacology Laboratory, Department of Pharmaceutics, Indian Institute of Technol- \\ ogy (Banaras Hindu University), Varanasi, India. \\ Email: shyam.chatterjee@web.de, *vikas.phe@itbhu.ac.in
}

Received May $9^{\text {th }}$, 2012; revised June $10^{\text {th }}, 2012$; accepted June $20^{\text {th }}, 2012$

\begin{abstract}
Consistent neglect of complex psychobiological processes involved in human health and diseases often leads to pharmacological discrepancies between preclinical and therapeutic observations. Numerous other pharmacological paradoxes arise from common practices of using dosing schedules in preclinical studies not identical to those in clinical studies. An Ayurveda centered holistic psychopharmacological strategy evolving from our efforts to identify plants potentially useful for preventing and combating illnesses and diseases could be a more rapid, rational, economically feasible, and realistic one for resolving such discrepancies and paradoxes. In this article, some observations made recently to experimentally very this conviction with the use of some well known medicinal plants are summarized and discussed in short. In addition, usefulness of the strategy for identifying edibles potentially useful for health care purposes, or for more objectively understanding “Ayurvedic biology” is pointed out.
\end{abstract}

Keywords: Mind-Body Medicine; Strategic Planning; Herbal Remedies; Preventive Pharmacology; Paradoxical Pharmacology

\section{Introduction}

Ayurveda is one of the oldest known holistic health care systems recommending diverse medicinal uses of plants for prevention and cure of diseases and illness [1]. During more recent decades many holistic principles, practices and strategies of Ayurveda have been well accepted by researchers and practitioners of numerous others interested in integrated, or more holistic, health care of patients. Hereupon, continued academic, commercial, and public interests in therapeutic potentials of Ayurvedic medicinal plants have played important roles. However, despite extensive efforts and considerable progress, precise definitions of medicinal phyto-chemistry and pharmacology of a vast majority of Ayurvedic plants are yet not possible in terms of theirs therapeutic potentials, uses and abuses. Such knowledge deficit is hampering not only more rational drug discovery and medicinal uses of Ayurvedic plants, but also more rapid progress towards Ayurveda centered integrative medicine. Although available information on medicinal phytochemistry and pharmacology of plant products are now widely used by many for drug discovery purposes, the strategies and experimental designs often used for such purposes are rarely

${ }^{*}$ Corresponding author. conceived or designed on holistic psychobiological principles of Ayurveda. This is mainly because modern experimental pharmacologists still continue to concentrate on pathological problems either of the body or of the brain, and define, or try to define, drugs and pharmacological targets in terms of their molecular chemistry only.

Such was not the case before 1970s when many currently widely used plant derived drugs and other health care products were discovered or identified by more holistic observations. During the second half of the $20^{\text {th }}$ century, most main stream researchers and practitioners of modern medicine became increasingly skeptical about therapeutic relevance of popular uses of herbal remedies and phyto-pharmaceuticals. This was not only due to high failures rates of many cost intensive and time consuming plant based drug discovery projects of pharmaceutical industries, but also due to inability of controlled clinical trials to obtain robust and reproducible clinical evidences for the medicinal values of a few more popular herbal remedies known in the western world. However, diverse medicinal uses of herbal remedies have continued to increase during these decades, and numbers of reports on fairly convincing and reliable preclinical pharmacological data on diverse types of plant products have also increased considerably. Since vast majorities of global 
population still continue to rely on herbal remedies for health care and medicinal purposes, novel paradigms and strategies for resolving discrepancies between preclinical and clinical observations are now being tried and proposed. However, most such proposals and practices pay little attention to the effective doses and treatment schedule commonly used in routine herbal practices.

Observations that chronic uses of some drugs can have effects opposite to those observed after their acute doses have led to the term "pharmacological paradox" for explaining several discrepancies between pharmacological observations and therapeutic benefits [2]. Efforts to resolve such paradoxes have led to the awareness of the complexities involved in biological functions of many well known pharmacological targets, and have also raised doubts about the validity of some pharmacological principles and practices often used for drug discovery and evaluation purposes [3]. Some of the numerous as yet unsolved pharmacological paradoxes are certainly those dealing with antidepressants and other psychoactive drugs. All antidepressants must be administered for at least several days, before their clinical effects can be observed, whereas many acutely acting psychotherapeutics lose their efficacy after repeated doses, or induce addiction dependence and other side effects.

During decades of extensive efforts in our laboratories to properly define psychopharmacological activity profiles of several well known medicinal herbs (like $\mathrm{Hy}$ pericum perforatum, Ginkgo biloba, Piper methysticum etc.) numerous discrepancies between observed effects after acute and repeated oral doses of their well standardized extracts in animal models were observed. These and diverse other pharmacological paradoxes revealed during such efforts, led us to a holistic pharmacological strategy for more rationally evaluating herbs for their therapeutic potentials [4]. In this strategy plant extracts (provisionally standardized according to existing recommendations and regulations, or specially prepared for experimental purposes) are orally administered to experimental animals, with or without experimentally induced pathologies, for two weeks before they are subjected to pharmacological studies. Choices of plants, their extracts, and doses, as well as animal models for such purposes are made by extensive ethno-pharmacological literature search. Hereupon special attention is paid to available information on structure activity relationships of all already known bioactive secondary plant metabolites and their other constituents. Using this strategy several novel therapeutic possibilities potentially offered by some Ayurvedic and other popularly known medicinal plants growing in India are now being reexamined in our laboratories. This communication summarizes some of our recent findings, and points out the advantages of the medicinal phytochemistry based holistic psychopharmacological strategy for reassessing therapeutic potentials of plants. In principle, this strategy is an extension of the classical ones commonly used during pre-genomic era for discovering drugs and therapeutic leads, whereupon emphasis is given to repeated dose studies during initial pharmacological studies. In general, such repeated dose studies are now conducted only before taking decisions on more time consuming and cost intensive preclinical safety and clinical trials, i.e. during the final stages of drug discovery projects.

\section{Rediscovering Holistic Pharmacology}

Ayurveda is the oldest known holistic health care system which emphasizes proper balance between the functions of body, mind, and soul is crucial for health maintenance. During more recent years, a somewhat more restricted definition of holistic medicine has been proposed [5], and a short history of clinical holistic medicine has appeared also [6]. Although no special terminology was used, pharmacological studies based on holistic principles have been practiced in India since long. Just one successful, and often cited, example of such efforts is reserpine (an alkaloid isolated from the Ayurvedic medicinal plant Rauwolfia serpentina). It became known to modern therapeutic researchers only after antihypertensive, psychopharmacological, and metabolic effects of the plant were identified in animal models. It was these findings only that eventually led to many postmodern psychopharmacological principles and concepts of currently practiced mind-body medicine in the western world.

Experimental strategies useful for holistic pharmacology were taught to us during 1990s by late Prof. Salil K. Bhattacharya of Banaras Hindu University. He was not only interested in popularizing experimental neuro-pharmacology in India, but also believed that sustainable therapeutic successes can be achieved only by proper maintenance of the balance between the mental health and metabolic function. The holistic strategy currently practiced in our laboratories is only one offshoot of numerous observations made during our collaborations with him. Hereupon those made with different types of $\mathrm{Hy}$ pericum perforatum extracts have been crucial. These efforts eventually led to the identification of Hyperforin as quantitatively the major antidepressant constituent of the plant. However, later on it was realized also that antidepressant like efficacy of its extracts must not necessarily contain hyperforin. Although some other polyphenolic isolated from the plant with antidepressants like activities in animal model could explain the discrepancy, they could not explain the entire spectrum of pharmacological activity profiles of different types of extracts obtainable from the plant.

Since pharmacological activity profiles of different 
types extracts of the plant (even though each one is well standardized according to commonly used modern definition of the word) were not always identical, it can be expected that their effects on human health will not be identical also. These observations suggested that theoretically it should be possible to design different types of extract from the same plant for different therapeutic uses. Analogous lesson has now also been learned by modern therapeutic researchers and drug discoverers. Consequently several drug discovery projects are now trying to find new therapeutic uses of older drugs $[7,8]$. It must be noted though, that a vast majority of findings indicating diverse therapeutic potentials of plants, or of known drugs and bioactive agents, come from in vitro studies, or from studies conducted acute doses of test agents. Therefore, more recently we reevaluated a commercially available standardized extract from Hypericum perforatum growing in India according to the postmodern concepts of psychobiology and pharmacological principles derived from those of modern holistic medical practices [9]. Activity spectrum of the extract revealed by such efforts [10-16] points out yet another novel therapeutic potential of the extract useful for helping patients suffering from, or prone to, diabesity, i.e. the spreading epidemic of the $21^{\text {st }}$ century [17].

It is now well recognized that reduced physical activity and inappropriate eating habits are two major environmental factors leading to diabesity $[18,19]$. To date, no universally acceptable, effective, safe, and affordable pharmaco-therapeutics or other measures for combating this global epidemic are available [20,21]. Therefore, we reevaluated some other readily available plants (with some similarities between their known pharmacological activity profiles and bioactive components with those of Hypericum perforatum) for their therapeutic potentials against diabesity. Only a few pharmacy students conducted these experiments, and available laboratory facilities of Banaras Hindu University were used for such purposes. These efforts revealed several novel pharmacological properties of Picrorhiza kurroa, Quassia amara, Fumaria indica, Piper longum; Andrographis paniculata, and some other traditionally known medicinal plants, which also are indicative of their therapeutic potentials against diverse spectrums of metabolic disorders or mental health problems often associated with diabesity [2244]. Since all novel pharmacological properties of the tested extracts obtained from such well scrutinized medicinal plants were observed after their well tolerated repeated daily oral doses, the possibility that these observations will have therapeutic implications can be considered as fairly high. Encouraged by these observations we are now evaluating the psychopharmacological activity profiles of a few commercially easily available secondary plant metabolites often encountered in such plants after their repeated daily doses. Observations made to date during such efforts have already revealed some unexpected psychopharmacological properties in a few such molecules. These observations could eventually be helpful in pharmacologically and analytically better standardize some medicinal plant extracts for therapeutic purposes.

\section{Holistic Pharmacology for Preventive Medicine}

The fact that prevention is better than cure is now well recognized by all medical practitioners and health care authorities around the globe, and it is well established also that at present prevention is the best possible option for combating the diabesity epidemic. Coexistence of obesity, or overweight, and type-2 diabetes is the primary diagnostic criterion for diabesity, which in principle has several similarities with the so called "metabolic syndrome”. Choices of appropriate fruits and vegetables are now highly recommended by nutritional therapists for prevention of this still expanding epidemic of modern civilization. These recommendations are analogous to those mentioned in Ayurveda for choices of food and eating habits. However, not all fruits and vegetables identified to date for such purposes are commonly available or affordable to a vast majority of Indian population, who according to WHO estimates are, and will continue to be, severely affected by the epidemic [45]. Unfortunately though, unlike in the western world very little efforts have yet been made to identify Indian vegetables that could be acceptable and affordable to its population for preventing further spread of the epidemic, or for helping patients with diabesity.

One potential food item identified to date for such purposes is mustard oil which is enriched in unsaturated fatty acids [46] and other bioactive phytochemicals. However, such oils cannot always be safely consumed in larger quantities, and they are neither cheap nor do they suit the taste of everyone. It is known though, that mustard (Brassica juncea) leaves (Hindi: “Sarson ka Saag”) are also fairly rich sources of other phytochemicals with known bioactivities potentially useful for combating several diabesity-associated comorbidities. Consequently, a Brassica juncea leaf extract containing some such phytochemicals was subjected to the holistic psychopharmacological screening battery identified during our recent ventures with traditionally known medicinal plants. As predictable from the phytochemical characteristics of the tested extract, it revealed a unique psychopharmacological activity profile in animals with experimentally induced hyperglycemia and hyperlipidemia, but had only minor effects on the quantified cognitive functions in healthy animals [47-49]. Some details of these and other 
earlier observations mentioned in this communication have already been reported, and others will be published soon. They have been mentioned here to point out only that appropriate uses of holistic animal behavioral models and repeated dose studies are useful and economically more feasible tools for diverse purposes, including those necessary for more objectively evaluating and discovering novel holistic health care possibilities offered by edibles and plants mentioned in Ayurveda. In any case, repeated daily dose effects with properly conceived plant extracts seems to be a useful means for more rationally integrating herbal remedies in the therapeutic armament of the modern practitioners of holistic medicine.

\section{Defining Holistic Psychopharmacology}

A recent "Google Scholar" search using the term "Holistic psychopharmacology" led to only one hit, and that too of a book reference [50], whereas that using the term "holistic neuro-pharmacology" gave none. Only two of the seven hits obtained by the use of the term "holistic pharmacology" were journal reports. One of them is certainly of interest to Ayurvedic researchers, and it used the term in an analogous sense to that used in our research group [51]. The other one used the expression "Systems (holistic) pharmacology" [52]. Although we wholeheartedly agree with the arguments and suggestions made in this report, it was disappointing to note that it completely neglect the complexities of the problems arising from repeated daily doses of drugs, or from effect of drugs on mental functions. Therefore, use of the word holistic only in brackets in this article could be considered as justifiable, or appropriate. It must be mentioned though, that all preclinical problems discussed in this report are valid for almost all herbal remedies as well. This repot could be a good starting point for younger researchers in their efforts to plan pharmacological studies for more objectively understanding traditionally known medicinal uses of plants.

Holism is a philosophical concept of the idea that the whole is greater than sum of its parts. As experimental pharmacologists we have used the term holistic psychopharmacology not only to point out the importance of integrated functions of mind body and soul in health and diseases, but also to stress the conviction that medicinal values of plants cannot be objectively defined by the concentrations of a few of their known constitutive molecules only. At present such knowledge is necessary though for more rational planning of experimental efforts and for avoiding inappropriate commercial uses, or other abuses, of herbal remedies. All medicinal ventures necessitate extraction of plants, and it is at this stage due considerations have to be given to the plant as a whole. This is also another lesson that has yet to be better learned (and prac- ticed by modern medicinal phytochemists!) from Ayurveda and other traditionally known medical systems.

As far as experimental pharmacology is concerned, it is important to remember that it is the feelings of the patients that ultimately decide the success or failures of all therapeutic and preventive measures, and that mental health condition and organ pathologies have, more often than not, a bidirectional relationship. Moreover, our current understanding on the web of obesity and diseases clearly point out the importance of society and other environmental factors in almost all major health problems [53]. Thus, experimental animal models useful for scrutinizing Ayurvedic remedies must be conceived to mimic human beings living in their societies. In this communication we have used the term "holistic psychopharmacology" only to point out, or remind, experimental pharmacologists that psychobiological processes are deeply involved in almost all medical sufferings. It is now becoming increasingly apparent that not only psychoactive drugs but also appropriate choices of plant food is helpful for leading a more happy, or satisfactory, life with reduced health risks $[54,55]$. Thus, scientifically better understanding the psychopharmacology of pleasurable, or healthy, vegetables and fruits could eventually not only lead to better preventive measures, but also could lead to knowhow necessary for more objectively understanding the concepts of “Ayurvedic biology”, or for discovering functionally novel therapeutics against diverse spectrums of comorbidities commonly encountered in aging population and in diabetic patients. More recent reports on curcumin [56,57], and some other edible phytochemicals, add experimental evidences to this conviction. It is disheartening though, to note that the effect of curcumin administered with curcuma powder cooked with our meals are seldom studied or reported. Socio-economic as well as medical importance of such studies cannot be overemphasized. If the curry could serve the purpose as efficiently as high doses of pure curcumin, preventive measures will be more economical and acceptable to patients.

\section{Concluding Remarks}

Draft of this manuscript was inspired by a recent thought leadership article in J-AIM [58], but we had to wait till the results of our before mentioned pharmacological venture with mustard leaves and some common bioactive components of medicinal plants. These were our very first ventures to use holistic pharmacology for identifying disease preventing edibles and for using the bio-active constituents of medicinal plant extracts. The observations made to date are encouraging, and reconfirm our conviction that available information on bioactivities of phytochemicals commonly consumed with food can also be a 
convenient starting point for identifying plants potentially useful for a desired medical condition. We are very thankful to the author of the leadership article for giving, precise, concise, and practicable hints and guides also useful for preclinical researchers interested in more objective understanding herbal remedies. One of the suggestions made by him was:

"The reverse pharmacology approach to drug evaluation, together with additional therapies based on the above holistic approach, could help restore recognition of Ayurveda's validity as a system of life and health science".

For such purposes holistic psychopharmacology, could be an economically more feasible and scientifically more acceptable approach for designing and conducting the necessary human studies. For example, having some hints on experimental pharmacology (and psychopharmacology) of edibles could be helpful for suggestions concerning proper choices of food and eating habits by medical practitioners. Some problems often confronted by many Ayurvedic practitioners deal with the banned or rarely available Ayurvedic plants, and yet others deal with authenticity and identity of Ayurvedic plants. A more reasonable resolution of numerous such problems can be more rapid, rational, and economically more feasible by the holistic strategy. Thus, like experimental archeology, experimental holistic pharmacology can be a useful means for better understanding and more rationally profiting from the teachings of Ayurveda. During all such ventures it must be kept in mind though, that at least more than 2000 years have elapsed since the origin of Ayurvedic teachings and that modern globalised Ayurveda is the resultant of many socioeconomic forces some of which has not been very reasonable [59].

Ethical problems, limitations, and drawbacks of animal pharmacology are well known and often controversially discussed. However, it cannot be ignored that advanced bio-technologies and other approaches currently widely used for drug discovery are often fairly cost intensive and more time consuming which eventually leads to higher therapy costs [60]. Considering this fact only, the holistic psychopharmacological strategy could be a promising starting point for better understanding the promiscuous pharmacology of medicinal plants and their bio-active constituents. In case this strategy could at least reduce the costs and failures of drug discovery and development efforts from plants and other natural products, pharmaceutical industries will no longer be miserly to have second thoughts on investing in plant based drug discovery projects. They will accept preclinical medical strategy or principles only after enough reliable and reproducible preclinical evidences have been generated by academic researchers. Without collaboration with an industry the holistic strategy now practiced in our laboratories would not have evolved. Therefore, we conclude that holistic psychopharmacological strategies could be useful at least for narrowing the conceptual gap between industrial and academic researchers on traditionally known medicinal values Ayurvedic and other plants.

\section{Acknowledgements}

Thanks are due to various Indian Government funding agencies and other scholarships to our students, without whose efforts we could not experimentally verify the holistic strategy under laboratory conditions available in the Banaras Hindu University.

\section{REFERENCES}

[1] K. S. Nagral, "Ayurved for Modern Medical Practitioners,” Chaukhamba Publishing House, New Delhi, 2008.

[2] R. A. Bond, "Is Paradoxical Pharmacology a Strategy Worth Pursuing?” Trends in Pharmacological Sciences, Vol. 22, No. 6, 2001, pp. 273-276. doi:10.1016/S0165-6147(00)01711-9

[3] C. Page, "Paradoxical Pharmacology: Turning Our Pharmacological Models Upside down," Trends in Pharmacological Sciences, Vol. 32, No. 4, 2011, pp. 197-200. doi:10.1016/j.tips.2011.02.006

[4] V. Kumar and S. S. Chatterjee, "Ethnopharmacology and Rational Evaluation of Herbal Remedies,” In: M. Eddouks, Ed., Handbook of Ethnopharmacology, Research Signpost, Research Signpost, Kerala, 2008, pp. 25-50.

[5] S. B. Kayne, "Introduction to Traditional Medicine," In: S. B. Kayne, Ed., Complementary and Alternative Medicine, 2nd Edition, Pharmaceutical Press, London, 2009, pp. 124.

[6] S. Ventegodt, I. Kandel and J. Merrick, “A Short History of Clinical Holistic Medicine,” The Scientific World Journal: TSW Holistic Health \& Medicine, Vol. 7, 2007, pp. 1622-1630. doi:10.1100/tsw.2007.238

[7] C. R. Chong and D. J. Sullivan, "New Uses for Old Drugs,” Nature, Vol. 448, No. 7154, 2007, pp. 645-646. doi:10.1038/448645a

[8] J. K. Aronson, “Old Drugs-New Uses,” British Journal of Clinical Pharmacology, Vol. 64, No. 5, 2007, pp. 563565. doi:10.1111/j.1365-2125.2007.03058.x

[9] K. Hamilton-West, "Psychobiological Processes in Health and Illness,” Sage Publications, Thousand Oaks, 2011.

[10] G. M. Husain, S. S. Chatterjee, P. N. Singh and V. Kumar, "Hypolipidemic and Antiobesity like Activity of Standardised Extract of Hypericum perforatum L. in Rats," ISRN Pharmacology, 2011, Article ID: 505247. doi: $10.5402 / 2011 / 505247$

[11] G. M. Husain, S. S. Chatterjee, P. N. Singh and V. Kumar, "Beneficial Effect of Hypericum perforatum on Depression and Anxiety in a Type 2 Diabetic Rat Model,” Acta Poloniae Pharmaceutica, Vol. 68, No. 6, 2011, pp. 913918.

[12] N. Kumar, G. M. Husain, P. N. Singh and V. Kumar, 
"Antiaggressive Activity of Hyperforin: A Preclinical Study,” Drug Discoveries \& Therapeutics, Vol. 3, No. 4, 2009, pp. 162-167.

[13] G. M. Husain, P. N. Singh and V. Kumar, "Beneficial Effects of a Standardized Hypericum perforatum Extract in Rats with Experimentally Induced Hyperglycemia," Drug Discoveries \& Therapeutics, Vol. 3, No. 5, 2009, pp. 215-220.

[14] S. Ineedi and V. Kumar, "Anti-Hyperlipidemic Activity of Hyperforin: An in Vivo Study in Rats," Pharmacologyonline, Vol. 2, 2009, pp. 133-139.

[15] G. M. Husain, S. S. Chatterjee, P. N. Singh and V. Kumar, "Antiaggressive Activity of Standaridised Extract of Indian Hypericum perforatum L,” Pharmacologyonline, Vol. 1, 2009, pp. 432-444.

[16] G. M. Husain, P. N. Singh and V. Kumar, “Anti-Diabetic Activity of Indian Hypericum perforatum L. on AlloxanInduced Diabetic Rats,” Pharmacologyonline, Vol. 3, 2008, pp. 889-894.

[17] Y. M. Farag and M. R. Gaballa, "Diabesity: An Overview of a rising Epidemic,” Nephrology Dialysis Transplantation, Vol. 26, No. 1, 2011, pp. 28-35. doi:10.1093/ndt/gfq576

[18] D. C. Lau, J. D. Douketis, K. M. Morrison, I. M. Hramiak, A. M. Sharma, E. Ur and Obesity Canada Clinical Practice Guidelines Expert Panel, “2006 Canadian Clinical Practice Guidelines on the Management and Prevention of Obesity in Adults and Children [Summary]," Canadian Medical Association Journal, Vol. 176, No. 8, 2007, pp. S1-S13. doi:10.1503/cmaj.061409

[19] National Institutes of Health, "Clinical Guidelines on the Identification, Evaluation, and Treatment of Overweight and Obesity in Adults-The Evidence Report," Obesity research, Vol. 6, No. S2, 1998, pp. 51S-209S.

[20] J. Ho, A. K. C. Leung and D. Rabi, "Hypoglycemic Agents in Management of Type 2 Diabetes Mellitus," Recent Patents on Endocrine, Metabolic \& Immune Drug Discovery, Vol. 5, No. 1, 2011, pp. 66-73.

[21] A. H. C. Wong, S. S. N. Barg and A. K. C. Leung, "Pharmacotherapy of Obesity," Recent Patents on Endocrine, Metabolic \& Immune Drug Discovery, Vol. 4, No. 1, 2010, pp. 47-58.

[22] V. Kumar, G. M. Husain and S. S. Chatterjee, "Search for Plants against Diabesity: A Comparative Preclinical Study," LAP-Lambert Academic Publishing AG \& Co. KG, Saarbrücken, 2011.

[23] G. M. Husain, P. N. Singh, R. K. Singh and V. Kumar, "Anti-Diabetic Activity of Standardised Extract of Quassia amara in Nicotinamide-Streptozotocin-Induced Diabetic Rats,” Phytotherapy Research, Vol. 25, No. 12, 2011, pp. 1806-1812.

[24] G. M. Husain, A. K. Thakur, P. N. Singh and V. Kumar, "Effect of Picrorhiza kurroa \& Quassia amara on High Fat Diet Induced Obesity in Rats," Proceedings of the Update Ayurveda 2010, Mumbai, 25-27 November, 2010, pp. 112-113.

[25] G. M. Husain, A. K. Thakur, P. N. Singh and V. Kumar, "Lack of Antidepressant and Anxiolytic Activity of
Picrorhiza kurroa and Quassia amara in Streptozotocin Induced Diabetic Rats,” Proceedings of the International Symposium on Brain Aging and Dementia: Basic and Translational Aspects, Varanasi, 29-30 November 2010, p. 58.

[26] G. M. Husain, P. N. Singh and V. Kumar, "Improvement of Cognitive Functions by Hypericum perforatum in Streptozotocin Induced Diabetic Rats," Proceedings of the International Symposium on Brain Aging and Dementia: Basic and Translational Aspects, Varanasi, 29-30 November 2010, p. 59.

[27] G. M. Husain, P. N. Singh, R. K. Singh and V. Kumar, "Effect of Hypericum perforatum, Picrohiza kurroa \& Quassia amara on Plasma Insulin Level in Nicotinamide-Streptozocin-Induced Diabetic Rats,” Proceedings of the 43rd Annual Conference of Indian Pharmacological Society \& International Conference on Pharmacology and Translational Research, Hyderabad, 13-16 December 2010, pp. S123-S124.

[28] G. M. Husain, P. N. Singh and V. Kumar, "Antidiabetic Activity of Standardized Extract of Picrorhiza kurroa in Rat Model of NIDDM,” Drug Discoveries \& Therapeutics, Vol. 3, No. 3, 2009, pp. 88-92.

[29] G. K. Singh and V. Kumar, "Acute and Sub-Chronic Toxicity Study of Standardized Extract of Fumaria indica in Rodents,” Journal of Ethnopharmacology, Vol. 134, No. 3, 2011, pp. 992-995. doi:10.1016/j.jep.2011.01.045

[30] G. K. Singh, S. K. Chauhan, G. Rai and V. Kumar, "Fumaria indica Is Safe during Chronic Toxicity and Cytotoxicity: A Preclinical Study,” Journal of Pharmacology \& Pharmacotherapeutics, Vol. 2, No. 3, 2011, pp. 191192. doi:10.4103/0976-500X.83287

[31] G. K. Singh and V. Kumar, "General Neuropharmacological Screening and Lack of Antidepressant Activity of Standardized Extract of Fumaria indica: A Preclinical Study," Electronic Journal of Pharmacology and Therapy, Vol. 3, 2010, pp. 19-28.

[32] G. K. Singh, R. Nageswar and V. Kumar, "Analgesic and Anti-Inflammatory Activity of Standardized Extract of Fumaria indica," Proceedings of the 62nd Indian Pharmaceutical Congress, Manipal, 17-19 December 2010, p. 107.

[33] G. K. Singh, A. Kumar and V. Kumar, "Fumaria indica Reverses Sodium Nitrite Induced Amnesia in Rodents," Proceedings of the Update Ayurveda 2010, Mumbai, 2527 November 2010, p. 110.

[34] G. K. Singh and V. Kumar, "Memory enhancing and AntiAmnestic Activity of Standardized Extract of Fumaria indica: An in-Vivo Study," Proceedings of the International Symposium on Brain Aging and Dementia: Basic and Translational Aspects, Varanasi, 29-30 November 2010, p. 75.

[35] G. K. Singh, A. Kumar and V. Kumar, "Fumaria indica Enhances Memory and Reverses Electroconvulsive Shock Induced Amnesia in Rodents," Proceedings of the International Symposium on Brain Aging and Dementia: Basic and Translational Aspects, Varanasi, 29-30 November 2010, p. 72.

[36] G. K. Singh and V. Kumar, "Beneficial Effects of Fu- 
maria indica in Mental Stress," Proceedings of the 5th Congress of Federation of Asian and Oceanian Neuroscience Societies and XXVIII Annual Meeting of Indian Academy of Neurosciences, Lucknow, 25-28 November 2010, p. 92.

[37] G. K. Singh and V. Kumar, "Anxiolytic Activity of Standardized Extract of Fumaria indica," Proceedings of the 27th Annual Conference of Indian Academy of Neurosciences, Jaipur, 18-20 December 2009, p. 81.

[38] G. K. Singh, G. Rai, S. S. Chatterjee and V. Kumar, "Anti-Aggressive, Brain Neurotransmitters and Receptor Binding Study of Fumaria indica in Rodents," Current Psychpharmacology, 2012, in press.

[39] G. K. Singh, G. Rai, S. S. Chatterjee and V. Kumar, "Potential Antianxiety Activity of Fumaria indica: A Preclinical Study,” Pharmacognosy Magazine, 2012, in press.

[40] G. K. Singh, G. Rai, S. S. Chatterjee and V. Kumar, "Beneficial Effects of Fumaria indica on Chronic StressInduced Neurobehavioral and Biochemical Perturbations in Rats," Chinese Medicine, Vol. 3, No. 1, 2012, pp. 4960. doi:10.4236/cm.2012.31009

[41] V. Kumar, G. K. Singh and G. Rai, "Biochemical Study of Fumaria indica," Proceedings of the12th International Congress of Ethnopharmacology-Traditional Medicines and Globalization-The Future of Ancient Systems of Medicine, Kolkata, 17-19 February 2012, p. 115.

[42] A. K. Thakur, S. S. Chatterjee and V. Kumar, "Potential of Andrographis panculata in Brain Disorders," Proceedings of the International Conference on Recent Advances in Pharmaceutical Science: Transferring Knowledge from Education to Practice, Kathmandu, 25-26 February 2012, pp. 31-32.

[43] S. Pokharel, J. S. Tripathi, S. K. Tiwari, S. S. Chatterjee and V. Kumar, "Perspectives of Piper longum L. in Neurological Disorders,” Proceedings of the International Conference on Recent Advances in Pharmaceutical Science: Transferring Knowledge from Education to Practice, Kathmandu, 25-26 February 2012, pp. 25-26.

[44] S. Ineedi, A. Shakya, G. K. Singh and V. Kumar, "Role of Hyperforin in Diabetes and Its Associated Hyperlipidemia in Rats," TANG: International Journal of Genuine Traditional Medicine, 2012, in press.

[45] J. Diamond “Medicine: Diabetes in India," Nature, Vol. 469, 2011, pp. 478-479. doi:10.1038/469478a

[46] T. Rastogi, K. S. Reddy, M. Vaz, D. Spiegelman, D. Prabhakaran, W. C. Willett, M. J. Stampfer and A. Ascherio, "Diet and Risk of Heart Disease in India," The American Journal of Clinical Nutrition, Vol. 79, No. 4, 2004, pp. 582-592.

[47] A. K. Thakur and V. Kumar, "Beneficial Effect of Brassica juncea in Co-Morbid Depression Associated with Alloxan Induced Diabetes in Rodents," Proceedings of the International Conference on Recent Advances in Pharmaceutical Sciences, Varanasi, 22-23 December 2010, p. 92.

[48] A. K. Thakur and V. Kumar, "Beneficial Effect of Brassica juncea in Co-Morbid Anxiety Associated with Alloxan Induced Diabetes in Rats," Proceedings of the 43rd Annual Conference of Indian Pharmacological Society \&
International Conference on Pharmacology and Translational Research, Hyderabad, 13-16 December 2010, p. S223.

[49] A. K. Thakur and V. Kumar, "Anti-Amnesic Activity of Brassica juncea in Diabetic Rats," Proceedings of the 12th International Congress of EthnopharmacologyTraditional Medicines and Globalization-The Future of Ancient Systems of Medicine, Kolkata, 17-19 February 2012, p. 116.

[50] E. Taylor, "The Mystery of Personality: A History of Psychodynamic Theories,” Springer, New York, 2009.

[51] R. H. Singh, "Exploring Large Evidence-Base for Contemporary Ayurveda," International Journal of Ayurveda Research, Vol. 1, No. 2, 2010, pp. 65-66. doi:10.4103/0974-7788.64394

[52] W. E. Stumpf, "Memo to the FDA and ICH: Appeal for in Vivo Drug Target Identification and Target Pharmacokinetics Recommendations for Improved Procedures and Requirements,” Drug Discovery Today, Vol. 12, No. 15-16, 2007, pp. 594-598. doi:10.1016/j.drudis.2007.06.006

[53] S. Kumanyika, R. W. Jeffery, A. Morabia, C. Rittenbaugh, V. J. Antipastis, and Public Health Approaches to the Prevention of Obesity (PHAPO) Working Group of the International Obesity Task Force (IOTF), "Obesity Prevention: The Case for Action,” International Journal of Obesity, Vol. 26, No. 3, 2002, pp. 425-436. doi:10.1038/sj.ijo.0801938

[54] F. Gomez-Pinilla, "Brain Food: The Effects of Nutrients on Brain Function,” Nature Reviews: Neuroscience, Vol. 9, No. 7, 2008, pp. 568-578. doi:10.1038/nrn2421

[55] D. O. Kennedy and E. L. Wightman, "Herbal Extracts and Phytochemicals: Plant Secondary Metabolites and the Enhancement of Human Brain Functions," Advances in Nutrition: An International Review, Vol. 2, No. 1, 2011, pp. 32-50.

[56] A. P. Kulkarni, D. A. Govender, G. J. Kotwal and L. A. Kellway, "Modulation of Anxiety Behavior by Intranasally Administered Vaccinia Virus Complement Control Protein and Curcumin in a Mouse Model of Alzheimer's Disease," Current Alzheimer Research, Vol. 8, No. 1, 2011, pp. 95-113.

[57] J. Chimakurthy and M. Talasila, "Effects of Curcumin on Pentylenetetrazole-Induced Anxiety-Like Behavior and Associated Changes in Cognition and Monoamine Levels,” Psychology \& Neuroscience, Vol. 3, No. 2, 2010, pp. 239-244. doi:10.3922/j.psns.2010.2.013

[58] R. H. Singh, "Exploring Issues in the Development of Ayurvedic Research Methodology,” Journal of Ayurveda and Integrative Medicine, Vol. 1, No. 2, 2010, pp. 91-95. doi:10.4103/0975-9476.65067

[59] D. Wujastyk and F. M. Smith, "Modern and Global Ayurveda: Pluralism and Paradigms," SUNY Press, New York, 2008.

[60] B. Patwardhan, "Traditional Medicine: Modern Approach for Affordable Global Health," Commission on Intellectual Property Rights IaPHC, World Health Organisation (WHO), Geneva, 2005. 